\title{
Pilot Study of Inhaled Aerosols Targeted via Magnetic Alignment of High Aspect Ratio Particles in Rabbits
}

\author{
Gillian E. S. Redman, ${ }^{1}$ Andrew R. Martin, ${ }^{1,2}$ Paul Waszak, ${ }^{3}$ Richard B. Thompson, ${ }^{4}$ \\ Po-Yin Cheung, ${ }^{3}$ Bernard Thébaud, ${ }^{3}$ and Warren H. Finlay ${ }^{1}$
}

${ }^{1}$ Department of Mechanical Engineering, University of Alberta, Edmonton, Canada T6G 2G8

${ }^{2}$ Air Liquide Centre de Recherche Claude-Delorme, 78354, Les Loges-en-Josas, France

${ }^{3}$ Department of Pediatrics, Faculty of Medicine and Dentistry, University of Alberta, Edmonton, Canada T6G $2 S 2$

${ }^{4}$ Department of Biomedical Engineering, University of Alberta, Edmonton, Canada T6G 2V2

Correspondence should be addressed to Gillian E. S. Redman, gillian.redman@gmail.com

Received 17 August 2010; Accepted 5 October 2010

Academic Editor: Xingmao Jiang

Copyright (C 2011 Gillian E. S. Redman et al. This is an open access article distributed under the Creative Commons Attribution License, which permits unrestricted use, distribution, and reproduction in any medium, provided the original work is properly cited.

Recently, inhaled pharmaceutical aerosols have seen increased investigation in the treatment of lung cancer, where the inability to deliver adequate therapeutic drug concentrations to tumour sites may be overcome with improved targeted delivery to the site of the tumour. In this study, the feasibility of magnetically targeted delivery of high aspect ratio particles loaded with iron oxide nanoparticles was studied in 19 New Zealand White rabbits. Half of the exposed rabbits had a magnetic field placed externally over their right lung. Iron sensitive magnetic resonance images of the lungs were acquired to determine the iron concentrations in the right and left lung of each animal. The right/left ratio increased in the middle and basal regions of the lung where, due to the morphology of the rabbit lung, this method of targeting is most effective. With further optimization, this technique could be an effective method for increasing the dose of drug delivered to a specific site within the lung.

\section{Introduction}

Pharmaceutical aerosols remain a growing field of interest in developing new methods of delivering therapeutic agents to the body. The large surface area of the lung offers excellent possibilities as a portal for fast and efficient drug delivery. Local drug delivery to the lung is common for the treatment of diseases such as asthma and chronic pulmonary infections. More recently, inhaled pharmaceuticals have attracted increased interest for the treatment of lung cancer [1-3]. One of the major obstacles in the treatment of lung cancer is the inability to deliver adequate therapeutic drug concentrations to the site of the tumour [1]. The use of inhaled chemotherapy in the treatment of lung cancer attempts to overcome this problem. Drug concentrations in the lung can be greatly increased without causing systemic toxicity, which results in greater drug concentrations at the site of interest and a decrease in the systemic side effects experienced by the patient [3]. The cytotoxic effect of the drug may result from direct topical application to the tumour, as well as absorption of the drug into the bronchial circulation which supplies blood to the tumour. In addition, tumours will often locally destroy the bronchial cilia, which in the case of aerosol delivery aids in retaining the drug at the desired site over a longer period of time [2]. However, these benefits could be further increased by better targeting delivery locally within the lung to the site of the tumour.

Indeed, there are a variety of methods to target a selected range of generations within the lung, including optimizing the particle density or size, or varying the inhalation flow rate [4-6]. However, these techniques are not able to target specific physical locations within the lung, which would be of interest for treatment of a disease such as lung cancer where often only one lobe of the lung is affected. Over recent years, there have been several different approaches to sitespecific targeted drug delivery. One of these methods utilizes 
an intratracheal nebulizing catheter to aerosolize the drug inside the targeted lobe or lung. Although this method has been shown to be effective in dogs, it is an invasive procedure with additional health risks associated with intubation of the subject [7]. In contrast, Dames et al. [8] have investigated a noninvasive method of magnetically targeted drug delivery in mice through the use of nebulized droplets containing superparamagnetic iron oxide nanoparticles. They found an increase in deposition of 7.9 times with the tip of an electromagnet centered $1 \mathrm{~mm}$ above the surgically exposed right lobe of the lung. Although this is a promising result, several complications could arise in attempting to apply this procedure for humans. Importantly, when moving the magnet tip to $5 \mathrm{~mm}$ away from the target site, the magnetic flux decreased by approximately 90\% [8]. Study into the design of magnetic fields for the purpose of magnetic drug targeting have shown that it remains feasible for superficial sites, but the rapid reduction in magnetic flux with distance from the source makes it a difficult method for targeting tissues deeper in the body without the use of surgically implanted ferromagnetic stents [9]. An alternative noninvasive approach to magnetic targeting in the lung is to utilize high aspect ratio particles (HARPs) in conjunction with superparamagnetic nanoparticles to target specific areas within the lung by controlling particle orientation with the use of a magnetic field [10-12].

The probability of deposition of HARPs is highly dependent on their orientation as they travel through the airways. HARPs will tend to align with their long axis parallel to the direction of flow, as seen in Figure 1(a), for which the probability of deposition is at its lowest [13]. As the tracheobronchial region transitions into the alveolar region, the dominant mechanism of deposition becomes interception, which occurs when the particle tip collides with the airway wall while the center of mass of the particle remains on a streamline. Airways are oriented in a random fashion within the lung. By aligning the HARPs in one direction, the particles would become randomly oriented with respect to the direction of flow in the airways, leading to a higher probability of deposition [14]. By loading HARPs with superparamagnetic nanoparticles, a preferred axis of magnetization is created along the length of the particle [10]. By placing a select region of the lung in a magnetic field, the orientation of the particles can be controlled by using the magnetic torque to align the long axis of the particles in the direction of the field, as seen in Figure 1(b). Martin and Finlay investigated the feasibility of this technique and found that the magnetic torque exerted on the particle was enough to overcome the shear forces of fluid flow in the lung for the range of particle aspect ratios of interest [10].

Using an airway model representative of the terminal bronchioles of the lung, Martin and Finlay showed an increase in deposition of cromoglycic acid (CA) HARPs of approximately 1.7 times with the application of a magnetic field compared to without [11]. The HARPs with and without magnetite had VMDs of 0.47 and $0.32 \mu \mathrm{m}$, respectively, and VMLs of 3.0 and $2.0 \mu \mathrm{m}$, respectively. Although (CA) is not intended for cancer therapy, it was chosen as a model drug, as it forms acicular crystals. The anticancer drug

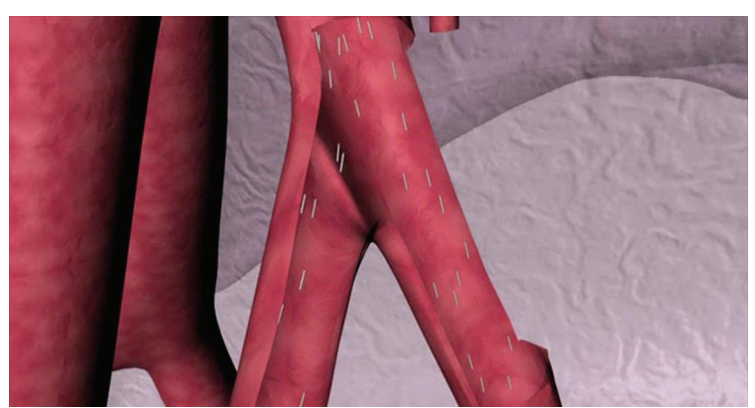

(a)

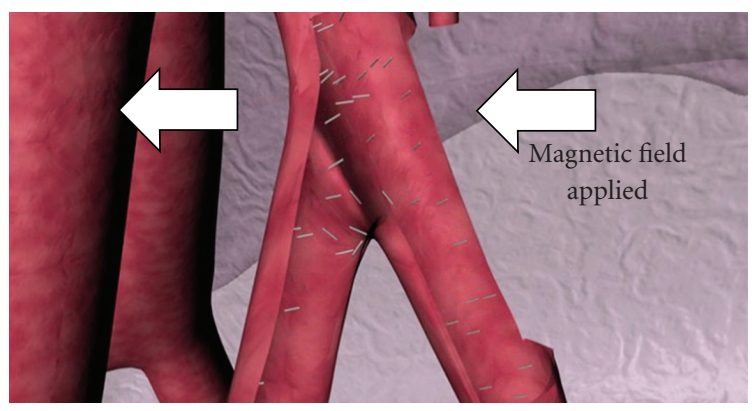

(b)

Figure 1: Alignment of HARPs in the lung (a) naturally (b) in the presence of a magnetic field.

paclitaxel has been loaded onto HARPs and shown to be more effective at shrinking tumours in this shape [15]. To further strengthen this positive result and the feasibility of this technique of magnetic targeting in the lung, an in vivo study was completed, as described herein. In the present study, New Zealand white rabbits were selected to determine the increase in aerosol deposition in a lung targeted with the application of a magnetic field. The use of laboratory animals for the study of inhaled particle deposition is common, as the total and regional particle deposition is qualitatively similar to humans [16]. The iron oxide nanoparticles also functioned as a magnetic resonance imaging (MRI) contrast agent, allowing the signal intensity of the images to be related to the concentration of iron [17]. This data was then used to calculate iron concentrations in the lungs of targeted and nontargeted rabbits, which were compared to show regional differences in deposition [18].

\section{Methods}

2.1. Aerosol Production. HARPs of cromoglycic acid (CA) were prepared following the crystallization method of Chan and Gonda [19] using cromolyn sodium salt purchased from Sigma-Aldrich Canada (Oakville, ON). To create one dose of suspension for nebulization, $10 \mathrm{mg}$ of CA was combined with $150 \mu \mathrm{L}$ of colloidal super paramagnetic iron oxide, FeREX (BioPAL, Worcester, MA), and $5 \mathrm{~mL}$ of deionized water. The FeREX had a concentration of $10 \mathrm{mg}$ Fe/ml. Dried CA particles and FeREX were dispersed by pipette to form an aqueous suspension prior to nebulization. These suspensions 


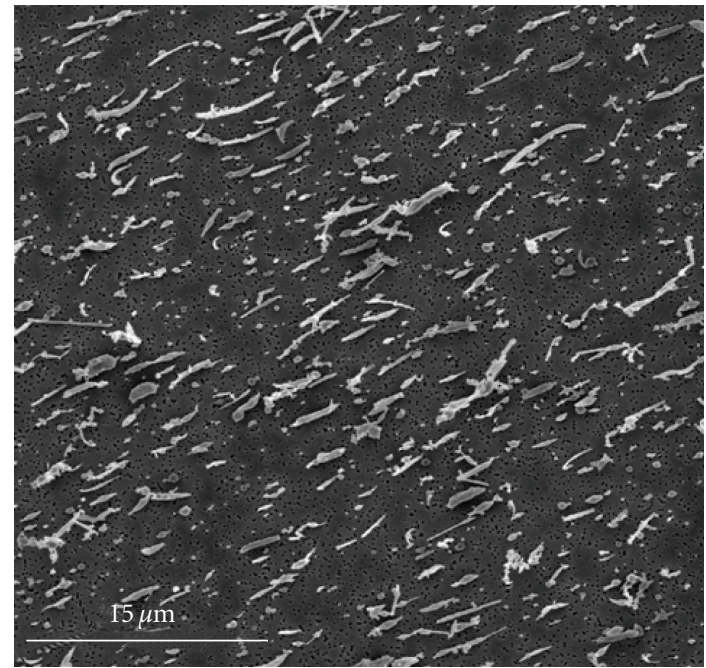

Figure 2: SEM image of high aspect ratio particles (HARPs) of CA and FeREX. The light-coloured aligned particles are the HARPs, and the small black dots are the pores of the membrane.

were nebulized using a clinically marketed vibrating mesh nebulizer (Aeroneb Pro; Aerogen, Galway, Ireland) and subsequently dried using a diffusion dryer containing silica gel (Component of Model 3475 Monodisperse Aerosol Generator; TSI, Shoreview, MN). This resulted in HARPs, similar to those previously created for an in vitro study, with diameters on the order of 500 nanometers and lengths varying from 1 to 10 micrometers [11]. The FeREX particles were specified to be in the range of $50-150 \mathrm{~nm}$ by the manufacturer. An SEM image taken of the HARPs generated can be seen in Figure 2. These HARPs were collected on a $0.2 \mu \mathrm{m}$ polycarbonate membrane (Isopore GTTP04700; Millipore, Billerica, MA) with a magnetic field produced across the face of the membrane to demonstrate alignment.

In order to ensure that regional lung distributions of iron are representative of regional distributions of CA throughout the lung, the distribution of iron across the range of HARP sizes was examined. This was done by drawing aerosol from the nebulizer and diffusion dryer through a cascade impactor (Mark II Andersen Impactor; Thermo Andersen, Smyrna, GA) at $28.3 \mathrm{l} / \mathrm{min}$. UV spectrophotometry was used to determine the masses of CA and iron deposited on each stage of the impactor. The mass of CA was found by washing with $0.01 \mathrm{~N}$ sodium hydroxide and assaying by UV spectrophotometry at a wavelength of $326 \mathrm{~nm}$. The mass of the iron was determined by methods similar to those described previously by Suwa et al. [20]. Prior to spectrophotometry at $468 \mathrm{~nm}$, the water insoluble FeREX was transformed to soluble $\mathrm{Fe}^{3+}$ using concentrated hydrochloric acid, and the CA was removed from suspension with the use of a $0.8 \mu \mathrm{m}$ filter.

2.2. Animals. This study involved 19 male New Zealand White Rabbits with weights ranging from 3.4 to $4.5 \mathrm{~kg}$. The animals were housed under conventional conditions under the guidelines of the Canadian Council on Animal Care

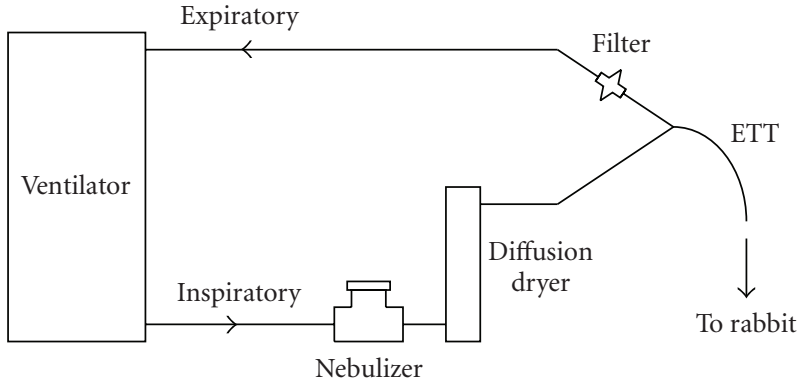

FIGURE 3: Schematic of mechanical ventilation circuit used to expose rabbits to aerosol. ETT: endotracheal tube.

for one week prior to the study. All animal procedures were approved by the University of Alberta Health Sciences Animal Policy and Welfare Committee.

2.3. Exposure. The nebulizer and diffusion dryer were added to the inspiratory line of a mechanical ventilation circuit (InfantStar; Infrasonics Inc., San Diego, CA) to deliver the aerosol to the lungs of ventilated rabbits. This circuit is outlined below in Figure 3 where the rabbit lung is ventilated via a $3.5 \mathrm{~mm}$ endotracheal tube. All excess aerosol (not deposited in the respiratory system of the rabbit) was collected on a filter (Respirgard; Vital Signs, Totowa, $\mathrm{NJ}$ ) in the expiratory line. After the animals were anesthetized using intravenous ketamine and xylazine infusions, a tracheostomy was performed, and a $3.5 \mathrm{~mm}$ endotracheal tube was inserted, connecting the rabbit to the mechanical ventilation circuit. The animals were then paralyzed using a pancuronium $(0.2 \mathrm{mg} / \mathrm{kg})$ intravenous infusion. All drug infusions were repeated as needed. Mechanical ventilation was performed using $100 \%$ oxygen with a peak inspiratory pressure of $14 \mathrm{~cm} \mathrm{H}_{2} \mathrm{O}$, a positive end-expiratory pressure of $2 \mathrm{~cm} \mathrm{H}_{2} \mathrm{O}$, a continuous flow of $6 \mathrm{l} / \mathrm{min}$, and an inspiratory time of $0.5 \mathrm{~s}$. The oxygen was neither warmed nor humidified prior to inspiration. Blood gases were monitored at interval throughout ventilation by arterial blood sampling and analyzed by ABL500 (Radiometer, Copenhagen, Denmark). To maintain an arterial $\mathrm{PCO}_{2}$ between $40 \pm 5 \mathrm{~mm} \mathrm{Hg}$, the breath frequency was adjusted between 20 and 25 breaths/min.

The animals were separated into three different categories of exposure: (1) no aerosol delivered, (2) aerosol delivered with no magnetic field applied, and (3) aerosol delivered with the application of a magnetic field. Three rabbits were mechanically ventilated for approximately 30 minutes but were not exposed to aerosol. Eight rabbits were exposed to two doses of the aerosol described above. Upon the completion of nebulization of the first dose, the reservoir in the nebulizer was refilled without opening the breathing circuit, and the second dose then ran to completion. The final eight rabbits were exposed to the aerosol but additionally had a magnetic field applied over the right lung. This was accomplished with the use of neodymium permanent magnets (two stacks of three, $2^{\prime \prime} \times 2^{\prime \prime} \times 0.5^{\prime \prime}$ magnets; Indigo Instruments Waterloo, ON). The two stacks of magnets were separated by $20 \mathrm{~cm}$ and positioned above and below the 
rabbit to create a field strength and gradient which decreased from approximately $35 \mathrm{mT}$ and $1.5 \mathrm{~T} / \mathrm{m}$, respectively, at the posterior side of the right lung to $20 \mathrm{mT}$ and $0.1 \mathrm{~T} / \mathrm{m}$ at the anterior side. In this position, magnetic field lines ran parallel to the direction of gravity, and the field gradient was directed so that small translational magnetic forces acting on particles augmented, rather than subtracted from, the force of gravity.

For all exposed rabbits ventilation lasted on average for approximately 45 minutes, with aerosol produced on average for approximately 23 minutes. All rabbits were euthanized with a pentobarbital intravenous infusion upon the completion of ventilation. The time between death and MRI scan was between 40 and 70 minutes for all rabbits.

2.4. Measurement of Deposition. MRI measurements were performed using a Siemens Sonata $1.5 \mathrm{~T}, 60 \mathrm{~cm}$ bore system (Siemens Medical Systems, Erlangen, Germany). Each rabbit was oriented in the prone position, in a $16 \mathrm{~cm}$ diameter volume birdcage coil, with the lungs placed at the centre of the coil to maximize reception sensitivity and uniformity. Iron oxide functions as an MRI contrast agent [17] which allows the signal intensity of the images to be related to the iron concentration. Specifically, the $T_{1}$ relaxation time, which describes the rate of recovery of the magnetization to thermal equilibrium, is directly related to the iron concentration according to the following relationship $[20,21]$ :

$$
\frac{1}{T_{1}}=\frac{1}{T_{1,0}}+\alpha \cdot c,
$$

where $T_{1,0}$ is the baseline relaxation time (determined from the 3 baseline rabbits), $c$ is the tissue concentration of iron, and $\alpha$ is a constant specific to the contrast agent as determined from previous work [18]. $T_{1}$ and $T_{1,0}$ values were calculated by inverting the MRI signal intensity and then fitting the magnitude of the recovered MRI signal intensity measured at increasing delays following the inversion preparation, as shown in

$$
\mathrm{SI}=\operatorname{abs}\left[\mathrm{SI}_{\infty}\left(1-k \cdot e^{-T_{i} / T_{1}}\right)\right],
$$

where SI is the signal intensity at inversion time $T_{i}$, and the constants $\mathrm{SI}_{\infty}, k$, and $T_{1}$ were determined by nonlinear regression.

To acquire the SI values, an inversion recovery fast spin echo pulse sequence with either 8 or $11 T_{i}$ times from 100 to $4000 \mathrm{~ms}$, in addition to the case of no inversion, was used to map the longitudinal relaxation time within the lung. The image parameters were as follows: $108 \times 25 \mathrm{~mm}$ field of view and a $448 \times 105$ matrix (in-plane spatial resolution interpolated to $250 \times 250 \mu \mathrm{m}$ ). There were $242.5 \mathrm{~mm}$ thick axial slices taken in two serial experiments (to prevent crosstalk between neighboring slices) which spanned the lung from the apex to the diaphragm. The pulse sequence had a repetition time, TR, of $5120 \mathrm{~ms}$ and an echo time, TE, of $12 \mathrm{~ms}$. The total acquisition time for each rabbit was approximately 30 minutes.

The images attained from the MRI scans were postprocessed to map iron concentrations in the right and left

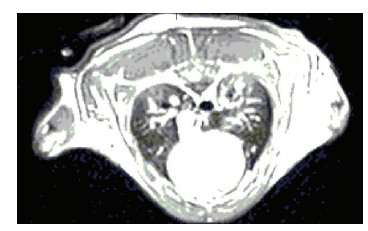

(a)

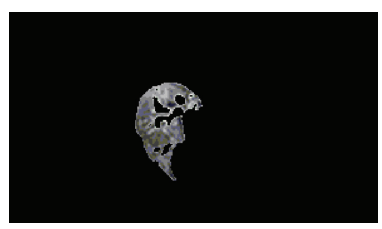

(c)

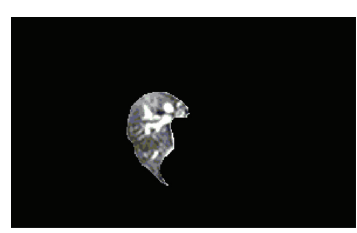

(b)

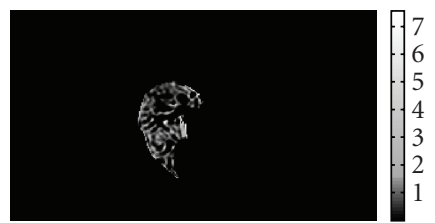

(d)
FIGURE 4: Sample of image processing: (a) original MRI image, (b) after selection of region of interest, (c) after removal of vasculature, and (d) iron concentrations in $\mu \mathrm{g} / \mathrm{cm}^{3}$.

lung for each rabbit. The lungs were manually segmented, and regions of high signal strength associated with lung vasculature were removed using a threshold filter as shown in Figure 4. A MATLAB (The Mathworks, Natick, MA) script was written to calculate the average $T_{1}$ relaxation times for user-selected regions of interest within the right and left lungs, in apical, medial, and basal subregions.

Using the regionally calculated $T_{1}$ and $T_{1,0}$ values, the iron concentrations were then compared between the right and left lung for each rabbit, globally, and in the apical, medial, and basal subregions. On average 18 of the $242.5 \mathrm{~mm}$ axial slices spanned the lung from top to bottom, in which case each subregion was comprised of 6 slices.

\section{Results}

Aerosol drawn through the cascade impactor showed a reasonably even distribution of iron oxide over the size range of CA particles created, ensuring that regional lung distributions of iron were representative of regional distributions of CA. This can be seen below in Figure 5 where statistically significant differences in the iron and CA-weighted size distributions were only seen for the plates 1,2 , and 3 .

For the three baseline rabbits an average $T_{1,0}$ relaxation time of $1531 \pm 45 \mathrm{~ms}$ was measured. The average wholelung $T_{1}$ relaxation time and iron concentration for the sixteen exposed rabbits were $1211 \mathrm{~ms}$ and $1.17 \pm 0.12 \mu \mathrm{g} / \mathrm{cm}^{3}$, respectively. The $T_{1}$ values and iron concentrations for both the left and right lungs of the exposed rabbits are shown in Table 1. The ratio of right to left lung iron concentration was $1.09 \pm 0.07$ in the nontargeted rabbits and $1.17 \pm$ 0.14 in the targeted rabbits. Nontargeted refers to rabbits exposed without the application of a magnetic field, and targeted refers to those with a magnetic field applied over the right lung.

The regional apical, medial, and basal analyses are summarized in Figure 5. It can be seen in Figure 6 that with application of the magnetic field, deposition in the targeted lung is decreased in the apical region and increased 
TABLE 1: Regional and total area-averaged $T_{1}$ relaxation times and iron concentrations for the left and right lung of nontargeted and targeted rabbits.

\begin{tabular}{|c|c|c|c|c|c|c|}
\hline \multirow{2}{*}{ Rabbit } & \multirow{2}{*}{ Region } & \multicolumn{2}{|c|}{ Left Lung } & \multicolumn{2}{|c|}{ Right Lung } & \multirow{2}{*}[\mathrm{Fe}]{$_{R} /[\mathrm{Fe}]_{L}$} \\
\hline & & $T_{1}[\mathrm{~ms}]$ & {$[\mathrm{Fe}]\left[\mu \mathrm{g} / \mathrm{cm}^{3}\right]$} & $T_{1}[\mathrm{~ms}]$ & {$[\mathrm{Fe}]\left[\mu \mathrm{g} / \mathrm{cm}^{3}\right]$} & \\
\hline \multirow{4}{*}{ Nontargeted } & Total & $1206 \pm 63$ & $0.61 \pm 0.28$ & $1200 \pm 60$ & $0.64 \pm 0.26$ & 1.09 \\
\hline & Apex & $1159 \pm 29$ & $0.84 \pm 0.15$ & $1140 \pm 34$ & $0.95 \pm 0.18$ & 1.22 \\
\hline & Middle & $1210 \pm 28$ & $0.59 \pm 0.13$ & $1193 \pm 24$ & $0.67 \pm 0.11$ & 1.30 \\
\hline & Base & $1205 \pm 32$ & $0.62 \pm 0.14$ & $1225 \pm 26$ & $0.52 \pm 0.11$ & 0.96 \\
\hline \multirow{4}{*}{ Targeted } & Total & $1224 \pm 44$ & $0.52 \pm 0.20$ & $1213 \pm 42$ & $0.57 \pm 0.18$ & 1.17 \\
\hline & Apex & $1178 \pm 20$ & $0.73 \pm 0.10$ & $1182 \pm 28$ & $0.72 \pm 0.13$ & 0.94 \\
\hline & Middle & $1233 \pm 20$ & $0.48 \pm 0.09$ & $1205 \pm 18$ & $0.60 \pm 0.08$ & 1.62 \\
\hline & Base & $1216 \pm 23$ & $0.56 \pm 0.11$ & $1211 \pm 19$ & $0.58 \pm 0.09$ & 1.18 \\
\hline
\end{tabular}

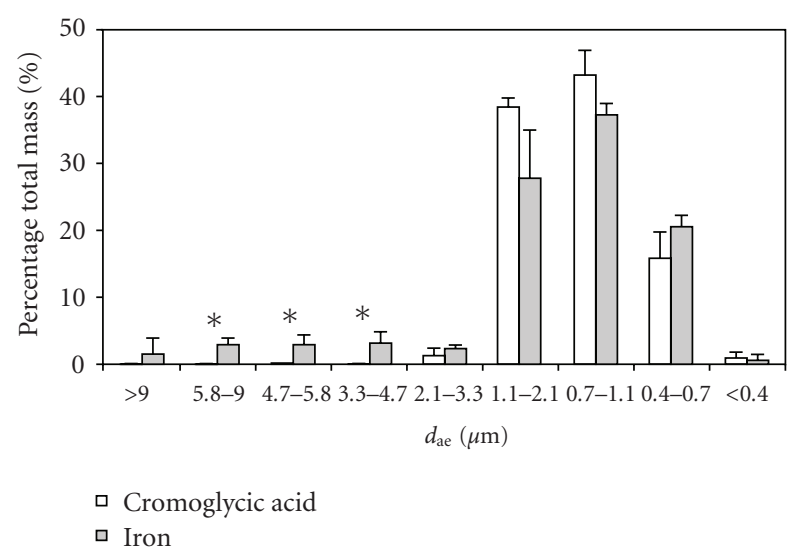

Figure 5: Aerodynamic diameter $\left(d_{\mathrm{ae}}\right)$ size distributions weighted by mass of iron and mass of cromoglycic acid, as determined by cascade impaction for the iron oxide-loaded cromoglycic acid aerosol. Asterisks indicate statistically significant differences between the two distributions $(P<.05$; two-tailed student's $t$-test for independent samples).

in the middle and basal regions. When comparing the iron concentration in the middle and base of the lung with the apex of the lung, it can be seen in Figure 7 that in the right lung of the targeted rabbits there is a targeted increase in deposition in the more basal regions.

\section{Discussion}

With the right lung being targeted, an increase in the ratio of the right to left lung iron concentrations was expected. After postprocessing the images from the 19 rabbits, it was found that the ratio of right/left iron concentration of the untargeted rabbits was similar to that of the targeted rabbits, which were 1.09 and 1.17 , respectively. However, examining the data in more detail, a larger increase in deposition in the middle and basal regions of the lung can be seen. In the middle region of the lung the ratio of right to left lung deposition increased from $1.30 \pm 0.13$ to $1.62 \pm 0.41$ and in the basal region deposition increased from $0.96 \pm 0.16$ to $1.18 \pm 0.17$.

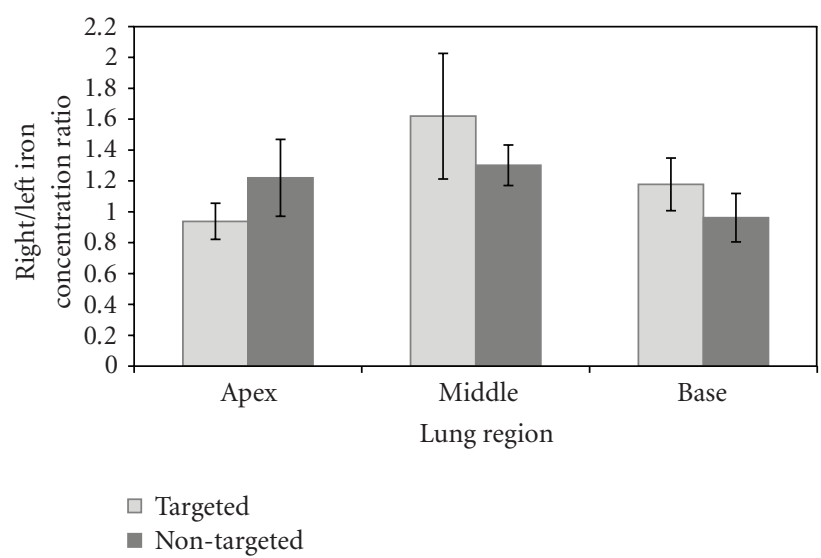

FIGURE 6: Ratios of regional right to left lung iron concentrations in targeted and nontargeted rabbits. Error bars represent standard errors of the mean.

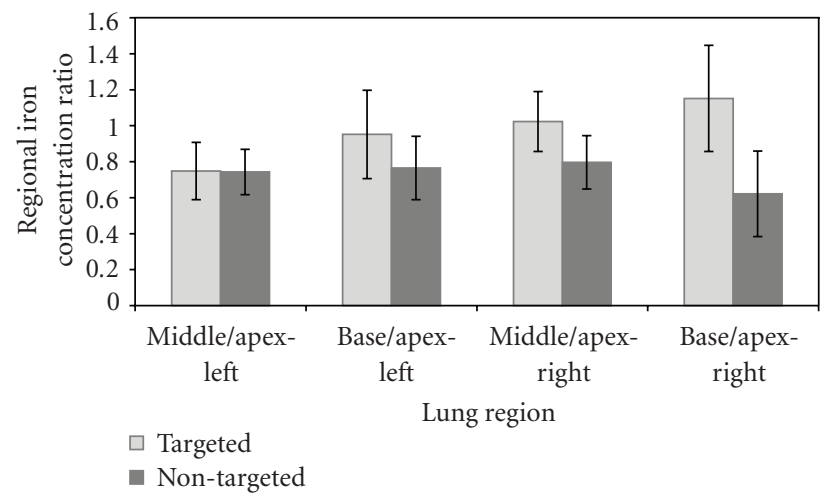

Figure 7: Ratios of iron concentration between middle and apical, and basal and apical regions of the left and right lungs of targeted and nontargeted rabbits. Error bars represent standard errors of the mean.

CA particles were loaded with iron oxide for the purpose of magnetic alignment, but also acted as an MRI contrast agent. As was seen in Figure 5, there were statistically significant differences in the iron and CA-weighted size distributions seen for plates 1, 2, and 3. However, this represents a small fraction of the overall mass and is likely 
due to diffusional losses of iron oxide nanoparticles not associated with the larger CA particles.

Although laboratory animals are commonly used for aerosol deposition studies, rodents such as the rabbit have a different lung morphometry than humans. In humans the airways branch dichotomously, meaning each parent airway divides into two equal sized daughter airways. The lung of the rabbit exhibits monopodial branching where multiple smaller daughter airways can branch off a single, longer parent airway [22]. In dichotomous branching, the distance a particle must travel from the trachea to the alveoli is similar for all the lobes whereas these distances can vary significantly in monopodial branching. The shortest distances occur in the upper lobes, which can result in these lobes experiencing higher concentrations of inhaled material for longer periods of time [23]. Thus, in the upper lobes, particles have a longer exposure time, increasing deposition by settling due to gravity; this mechanism likely dominates the probability of deposition over interception. Therefore, it is possible that this would result in a minimal effect observed from alignment of the HARPs in the upper region of the lung. This hypothesis is further supported by the higher total iron concentrations in the apical region of the lung compared with the middle and basal regions. This is seen in Figure 7 where the ratios of middle/apex and base/apex for the right and left lungs are all less than one for the untargeted rabbits. Despite the decreased efficiency of targeting in the apex, the effect of targeting is seen in the middle and basal regions of the lung where interception is expected to have a larger impact on the probability of deposition. In the apical region, iron concentrations were decreased with the application of the magnetic field. The method used to divide the lungs into three regions was rather crude and may have resulted in some of the conducting airways of the apex being incorrectly classified as part of the middle region. Targeting is expected to increase deposition in these incorrectly classified conducting apical airways, decreasing the total aerosol that would continue for possible deposition into the apically classified region. This would result in a perceived decrease in deposition in the apical region. A more detailed and accurate method of separating the regions of the lung, similar to the computer models of lung morphology used in conjunction with single photon emission computed tomography (SPECT) images by Schroeter et al. [24], would provide valuable data regarding regional deposition.

It is important to note that these results were not statistically significant $(P>.05)$. For example, when performing an unpaired two-tailed student's $t$-test for independent samples on the data from Figure 7, the $P$ values were found to be .98 and .55 in the middle and basal regions of the untargeted left lung, respectively. In the middle and basal regions of the targeted right lung, these $P$ values were found to decrease to .33 and .18, respectively. Statistical significance could potentially be obtained by increasing the number of subjects. However, a more desirable solution would be to decrease variability in the experimental methodology by eliminating some of the limitations present in this study.

A number of these limitations are as follows.
Within the study group, consisting of 19 subjects, considerable intersubject variability existed. This variability, in conjunction with the small number of subjects, led to results which were not statistically significant, although the data shows promise. Specifically, the baseline relaxation time $\left(T_{1,0}\right)$ determined from the three baseline rabbits varied from 1249 to $1444 \mathrm{~ms}$. This value, representing the properties of the lung tissue without the presence of iron, is critical in determining the iron concentration of the lungs in the exposed rabbits, and our use of an average baseline relaxation time, rather than subject specific times, limits the discriminating power of the present study. Specifically, iron concentrations were determined from variation in $T_{1}$ times only as averages for each group; mapping of iron concentrations in individual rabbits would be possible only with individual $T_{1,0}$ baselines values.

Additionally, the ventilator used in this study did not measure the inhalation tidal volumes, eliminating the possibility of determining the total deposition fraction. If the majority of the aerosol entering the respiratory system deposits without the presence of a magnetic field, any further increase in deposition with the application of a magnetic field is limited. The results of this limitation would be a decrease in observed effectiveness of the targeting.

In moving forward with this study, the limitations of intersubject variability could be overcome by increasing the number of subjects, obtaining individual baselines for each subject, or increasing the effectiveness of the targeting. By measuring the individual baseline relaxation time $\left(T_{1,0}\right)$ for each subject, a much more accurate estimate of iron concentration would be calculated in each animal. This would require an MRI scan to be taken of each subject before and after aerosol exposure, which would allow each rabbit to be its own baseline. Increasing the effectiveness of the targeting through optimization of the HARPs could further help in overcoming intersubject variability. The probability of deposition with the application of the magnetic field will be increased by increasing the length of the HARPs. Alignment of longer particles with an external magnetic field would further increase interception with the airway walls [11].

\section{Conclusion}

This pilot study showed an increase in deposition of high aspect ratio particles (HARPs) of cromoglycic acid (CA) loaded with iron oxide nanoparticles with the application of a magnetic field in the middle and basal regions of the lung. As interception is not the dominant mechanism of particle deposition in the apex of rabbits, minimal effect was seen in the apical region. Further work must be done to decrease the effect of the intersubject variability through the use of individual baseline relaxation times $\left(T_{10}\right)$ and optimized HARP lengths to yield results that will be statistically significant. With further optimization of this technique, and the delivery system, it may be an effective method for the treatment of diseases such as lung cancer by increasing the dose of drug delivered to a specific site within the lung. 


\section{Acknowledgments}

This paper was funded in part by an NSERC Special Research Opportunity grant. Kelvin Chow is gratefully acknowledged for supplying the MATLAB script to filter out vasculature.

\section{References}

[1] G. A. Otterson, M. A. Villalona-Calero, S. Sharma et al., "Phase I study of inhaled doxorubicin for patients with metastatic tumors to the lungs," Clinical Cancer Research, vol. 13, no. 4, pp. 1246-1252, 2007.

[2] T. Tatsumura, S. Koyama, M. Tsujimoto, M. Kitagawa, and S. Kagamimori, "Further study of nebulisation chemotherapy, a new chemotherapeutic method in the treatment of lung carcinomas: fundamental and clinical," British Journal of Cancer, vol. 68, no. 6, pp. 1146-1149, 1993.

[3] S. Sharma, D. White, A. R. Imondi, M. E. Placke, and D. M. Vail, "Development of inhalational agents for oncologic use," Journal of Clinical Oncology, vol. 19, no. 6, pp. 1839-1847, 2001.

[4] W. H. Finlay, The Mechanics of Inhaled Pharmaceutical Aerosols: An Introduction, Academic Press, London, UK, 2001.

[5] D. A. Edwards, J. Hanes, G. Caponetti et al., "Large porous particles for pulmonary drug delivery," Science, vol. 276, no. 5320, pp. 1868-1871, 1997.

[6] P. Camner, M. Anderson, K. Philipson et al., "Human bronchiolar deposition and retention of 6-, 8-, and $10-\mu \mathrm{m}$ particles," Experimental Lung Research, vol. 23, no. 6, pp. 517 535, 1997.

[7] K. Selting, J. C. Waldrep, C. Reinero et al., "Feasibility and safety of targeted cisplatin delivery to a select lung lobe in dogs via the AeroProbe intracorporeal nebulization catheter," Journal of Aerosol Medicine and Pulmonary Drug Delivery, vol. 21, no. 3, pp. 255-268, 2008.

[8] P. Dames, B. Gleich, A. Flemmer et al., "Targeted delivery of magnetic aerosol droplets to the lung," Nature Nanotechnology, vol. 2, no. 8, pp. 495-499, 2007.

[9] B. Gleich, N. Hellwig, H. Bridell et al., "Design and evaluation of magnetic fields for nanoparticle drug targeting in cancer," IEEE Transactions on Nanotechnology, vol. 6, no. 2, pp. 164169, 2007.

[10] A. R. Martin and W. H. Finlay, "Magnetic alignment of aerosol particles for targeted pulmonary drug delivery: comparison of magnetic and aerodynamic torques," Journal of Computational and Theoretical Nanoscience, vol. 5, no. 10, pp. 2067-2070, 2008.

[11] A. R. Martin and W. H. Finlay, "Enhanced deposition of high aspect ratio aerosols in small airway bifurcations using magnetic field alignment," Journal of Aerosol Science, vol. 39, no. 8, pp. 679-690, 2008.

[12] A. R. Martin and W. H. Finlay, "Alignment of magnetiteloaded high aspect ratio aerosol drug particles with magnetic fields," Aerosol Science and Technology, vol. 42, pp. 295-298, 2008.

[13] T. Myojo, "Deposition of fibrous aerosol in model bifurcating tubes," Journal of Aerosol Science, vol. 18, no. 3, pp. 337-347, 1987.

[14] I. Báláshazy, T. B. Martonen, and W. Hofmann, "Fiber deposition in airway bifurcations," Journal of Aerosol Medicine, vol. 3, pp. 243-260, 1990.
[15] Y. Geng, P. Dalhaimer, S. Cai et al., "Shape effects of filaments versus spherical particles in flow and drug delivery," Nature Nanotechnology, vol. 2, no. 4, pp. 249-255, 2007.

[16] R. F. Phalen, M. J. Oldham, and R. K. Wolff, "The relevance of animal models for aerosol studies," Journal of Aerosol Medicine and Pulmonary Drug Delivery, vol. 21, no. 1, pp. 113-124, 2008.

[17] M.-F. Bellin, "MR contrast agents, the old and the new," European Journal of Radiology, vol. 60, no. 3, pp. 314-323, 2006.

[18] A. R. Martin, R. B. Thompson, and W. H. Finlay, "MRI measurement of regional lung deposition in mice exposed noseonly to nebulized superparamagnetic iron oxide nanoparticles," Journal of Aerosol Medicine and Pulmonary Drug Delivery, vol. 21, no. 4, pp. 335-341, 2008.

[19] H.-K. Chan and I. Gonda, "Aerodynamic properties of elongated particles of cromoglycic acid," Journal of Aerosol Science, vol. 20, no. 2, pp. 157-168, 1989.

[20] T. Suwa, S. Ozawa, M. Ueda, N. Ando, and M. Kitajima, "Magnetic resonance imaging of esophageal squamous cell carcinoma using magnetite particles coated with antiepidermal growth factor receptor antibody," International Journal of Cancer, vol. 75, no. 4, pp. 626-634, 1998.

[21] S. Gohr-Rosenthal, H. Schmitt-Willich, W. Ebert, and J. Conrad, "The demonstration of human tumors on nude mice using gadolinium-labelled monoclonal antibodies for magnetic resonance imaging," Investigative Radiology, vol. 28, no. 9, pp. 789-795, 1993.

[22] R. B. Schlesinger and L. A. McFadden, "Comparative morphometry of the upper bronchial tree in six mammalian species," Anatomical Record, vol. 199, no. 1, pp. 99-108, 1981.

[23] F. J. Miller, R. R. Mercer, and J. D. Crapo, "Lower respiratory tract structure of laboratory animals and humans: dosimetry implications," Aerosol Science and Technology, vol. 18, no. 3, pp. 257-271, 1993.

[24] J. D. Schroeter, J. S. Fleming, D. Hwang, and T. B. Martonen, "A computer model of lung morphology to analyze SPECT images," Computerized Medical Imaging and Graphics, vol. 26, no. 4, pp. 237-246, 2002. 

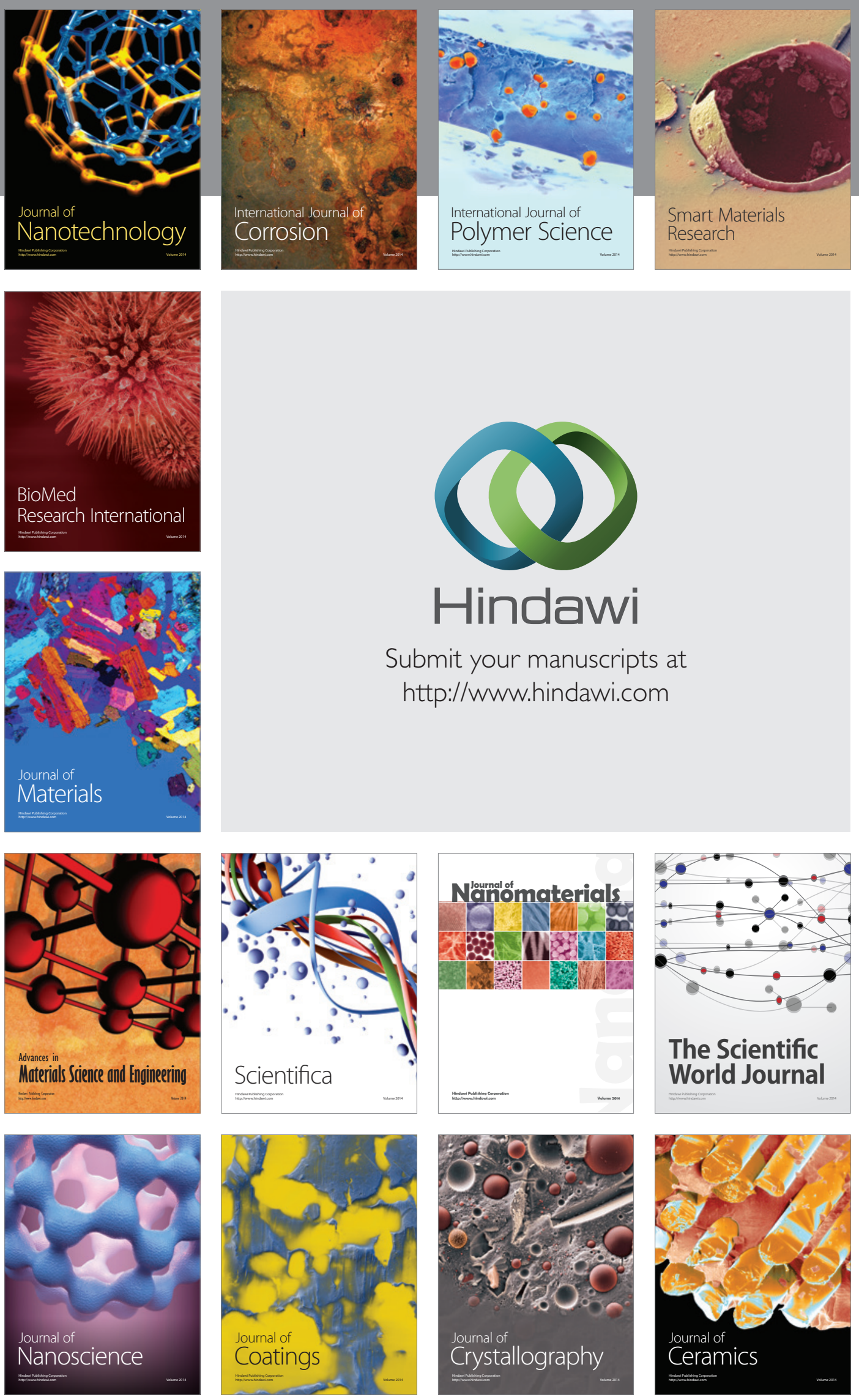

The Scientific World Journal

Submit your manuscripts at

http://www.hindawi.com

\section{World Journal}

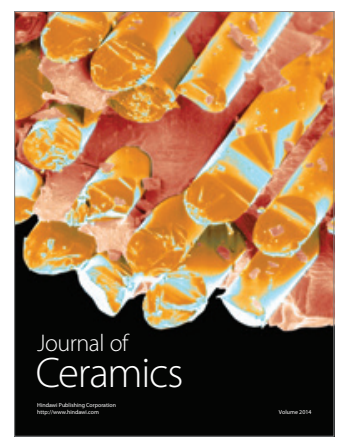

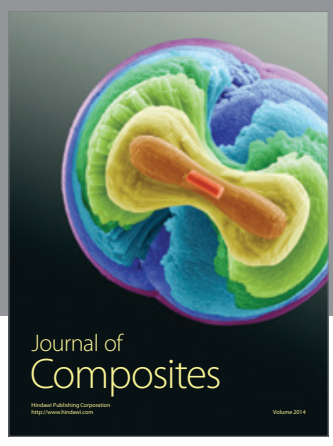
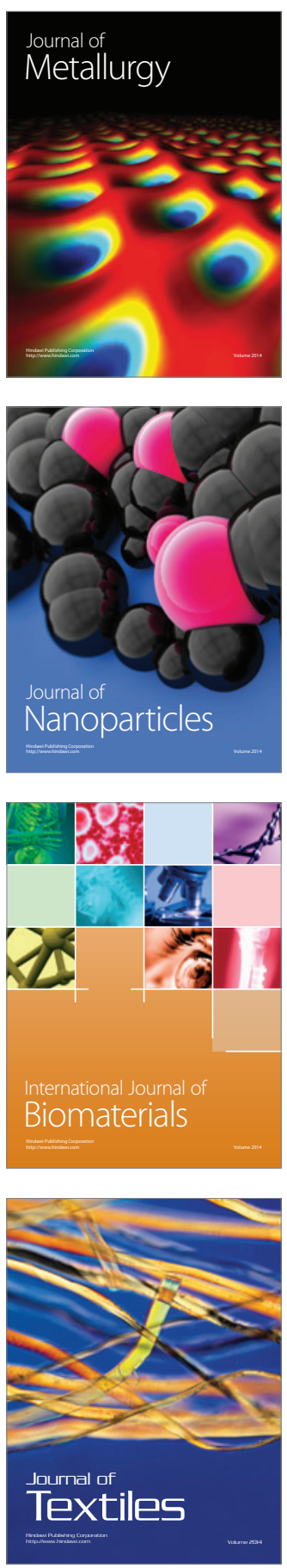